DOI: $10.3901 / J M E .2020 .17 .233$

\title{
直接驱动型压电式喷头微滴产生过程 数值模拟及试验研究"
}

\author{
肖 渊 ${ }^{1,2}$ 张威 ${ }^{1}$ 王 盼 $^{1}$ 李红英 ${ }^{1}$
}

(1. 西安工程大学机电工程学院 西安 710048;

2. 西安市现代智能纺织装备重点实验室 西安 710600)

\begin{abstract}
摘要: 针对目前挤压管型压电式喷头结构复杂、组装工艺要求高及价格昂贵等问题, 设计一种结构简化的环形压电陶瓷直接 驱动喷射材料的微滴喷射装置。通过建立多物理场耦合压电式喷头微滴喷射两相流模型, 研究驱动电压、流体黏度及表面张 力等参数对微滴成形动力学行为的影响。在此基础上, 利用构建的压电式喷头进行微滴喷射试验。结果表明: 驱动电压幅值 与产生微滴的速度及体积大小呈线性关系, 喷射材料黏度增大产生的微滴喷射速度及体积减小, 表面张力直接影响液滴形成 过程的喷射材料液体丝带长度及断裂距离; 采用喷嘴孔径为 $65 \mu \mathrm{m}$ 的喷头, 在稳定喷射条件下, 可按需产生直径约 $80 \mu \mathrm{m}$ 的均匀微滴, 验证了设计的直接驱动式压电喷头按需产生均匀微滴的可行性。
\end{abstract}

关键词: 微滴喷射; 直接驱动; 压电式喷头; 多物理场; 数值模拟

中图分类号: TS852

\section{Numerical Simulation and Experimental Research of Micro-droplet Generation by Directly Actuated Piezoelectric Nozzle}

\author{
XIAO Yuan ${ }^{1,2}$ ZHANG Wei ${ }^{1}$ WANG Pan ${ }^{1}$ LI Hongying ${ }^{1}$ \\ (1. College of Mechanical and Electrical Engineering, Xi'an Polytechnic University, Xi'an 710048; \\ 2. Xi'an Key Laboratory of Modern Intelligent Textile Equipment, Xi'an 710600)
}

\begin{abstract}
A micro-droplet generator with simplified structure by ring piezoelectric ceramic directly driving the ejection material is constructed to solve the problem of the complicated structure, high assembly process requirements and high price caused by squeezed piezo-type nozzle. The effects of driving voltage, fluid viscosity, surface tension and other parameters on the dynamic behavior of micro-droplet forming is studied by establishing a two-phase flow model of piezoelectric nozzle micro-droplet ejection by multi-physics coupling. Based on this, the droplet ejection test has performed using the constructed piezoelectric nozzle. The results show that driving voltage amplitude has a linear relationship with the velocity and volume of droplet production. The ejection velocity and volume of droplet produced decrease with the increase of ejection material viscosity, and the surface tension directly affects the length of the liquid ribbon of the jetting material during the droplet formation and fracture distance. Under the stable ejection conditions, a nozzle with a diameter of $65 \mu \mathrm{m}$ can produce uniform droplets of about $80 \mu \mathrm{m}$ in diameter as required, which verifies the feasibility of the designed directly driven piezoelectric nozzle to generate uniform micro-droplets on demand.
\end{abstract}

Key words: micro-droplet ejection; directly driving; piezoelectric nozzle; multi-physics; numerical simulation

\section{0 前言}

微滴喷射技术是一种新型快速成形制造技术， 通过将具有特定功能的流体材料精确沉积到基板 上，逐点、逐层堆积实现不同几何形状零件的快速

\footnotetext{
* 国家自然科学基金和西安市现代智能纺织装备重点实验室项目 (2019220614SYS021CG043)资助项目(51475350)。20191204 收到初稿, 20200311 收到修改稿
}

成形。该技术具有成本低、效率高、成形材料广、 非接触等优点, 是一项颇具发展潜力的材料成形新 技术 ${ }^{[1]}$, 因而在生物工程、微电子封装、柔性电路 成形及微机电系统等领域具有广阔的应用前景 ${ }^{[2-6]}$ 。

目前常见的微滴产生驱动方式有微阀、热气泡、 压电、静电及气压驱动等 ${ }^{[7-9]}$ 。压电式微滴喷射装置通 过压电元件产生的振动, 实现微滴的按需可控喷射, 具有响应频率高, 喷射精度高, 不易受外界电磁场干 扰等优点, 得到了广泛的应用 ${ }^{[10]}$ 。按照压电元件的变 
形方式, 常见的压电式喷头结构有撞击式、弯曲式、 剪切式及挤压式等 ${ }^{[11]}$ 。其中挤压式结构由环形压电元 件通过粘合剂紧贴于毛细玻璃管外壁构成, 结构简单, 与墨水接触面积大, 可实现较高黏度材料的打印, 但 目前的挤压管型压电式喷头存在结构复杂、组装工艺 要求高及价格昂贵等问题。基于此, 本文设计了一种 压电元件直接驱动喷射材料的结构形式, 并对微滴的 喷射机理及喷射过程影响因素进行详细研究。

目前, 国内外针对挤压管型压电式喷头的微滴成 形过程进行了深入的研究, 文献[12]基于平面应变假 设, 建立了挤压管型压电式喷嘴的静力学模型, 得到 了压电陶瓷管在径向电场作用下形变表达式。文献[13] 采用数值分析的方法, 对不同驱动电压下挤压式喷头 的墨滴成形过程进行研究。文献[14-15]提出将传感器 集成到挤压式喷墨头中的思路, 并建立多物理场挤压 式喷墨头的仿真模型, 研究了容腔内压力和液滴体积 间的关系。上述研究过程均采用压电元件间接驱动喷 射材料的方式实现微滴的喷射, 与直接驱动式的作用 机理不尽相同, 因此需要对直接驱动方式下压电元件 形变规律、微滴产生过程及材料属性等对微滴成形过 程的影响规律进行深入研究。基于此, 本文构建了直 接驱动型压电式微滴喷射装置, 建立了多物理场耦合 的压电式微滴喷射两相流模型, 研究驱动电压、液体 黏度及表面张力等因素对成形微滴的影响。在此基础 上, 利用构建的微滴喷射试验系统, 实现直接驱动型 压电式喷头的按需喷射试验, 以验证该结构喷头产生 均匀微滴的可行性。

\section{1 直接驱动型喷头结构及工作原理}

\section{1 喷头结构模型}

设计的直接驱动型压电式喷头主要由喷嘴、储 液腔及环形压电陶瓷驱动元件组成, 如图 1 所示。 其中喷嘴长度 $l$ 为 $3 \mathrm{~mm}$ 、喷嘴口直径 $D_{4}$ 为 $100 \mu \mathrm{m}$,

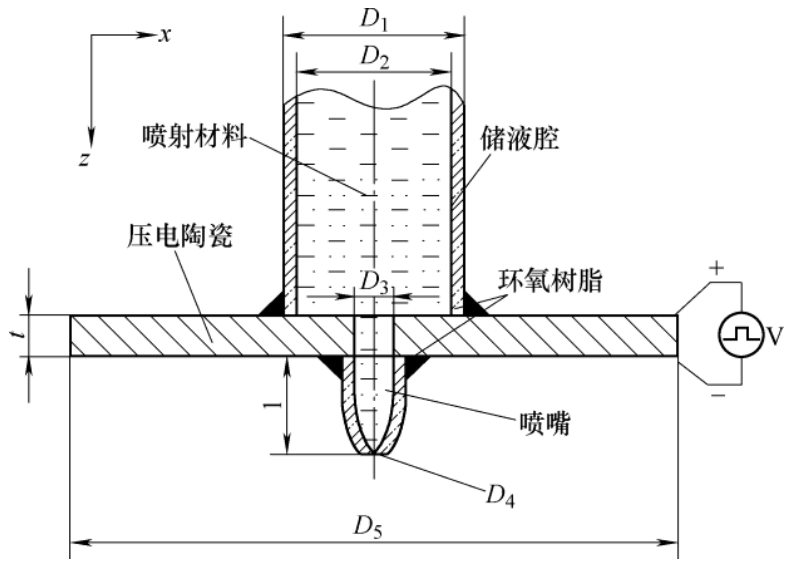

图 1 直接驱动型压电式喷头结构图
通过玻璃圆管拉制工艺制作，呈雉形流线型; 储液 腔外径 $D_{1}$ 为 $8 \mathrm{~mm}$ 、内径 $D_{2}$ 为 $6 \mathrm{~mm}$, 用于喷射材 料的储存, 由玻璃管制成; 环形压电陶瓷选用保定 市宏声声学电子器材有限公司的 PZT-5 材料的陶 瓷, 内径 $D_{3}$ 为 $2 \mathrm{~mm}$ 、外径 $D_{5}$ 为 $20 \mathrm{~mm}$ 、厚度 $t$ 为 $1 \mathrm{~mm}$, 压电陶瓷内壁直接与喷射材料接触, 内壁 产生的变形将直接作用于喷射材料。喷嘴、储液腔及 环形压电陶瓷间通过环氧树脂粘合为一整体结构。

\section{2 喷头的工作原理}

环形压电陶瓷在驱动电压的激励下内壁产生形 变, 直接挤压喷射材料, 驱动液体从喷嘴喷射, 形 成微滴, 其喷射过程如图 2 所示。

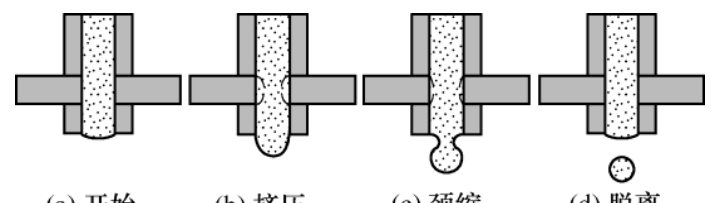
(a) 开始
(b) 挤压
(c) 颈缩
(d) 脱离

图 2 微滴喷射成形过程

图 2 中, 环形压电陶瓷内壁直接与喷射材料接 触, 在驱动电压的作用下压电陶瓷内壁产生的径向 位移直接挤压喷射材料, 推动其在喷嘴口形成具有 一定速度的液柱。当驱动信号作用完成后, 压电陶 瓷变形恢复, 腔体内压力减小, 液柱尾部开始收缩, 而液柱前端由于惯性继续向下运动, 两种运动使得 液柱尾部产生颈缩, 随着颈缩加剧, 液柱发生断裂, 在表面张力和惯性的共同作用下呈近似球形飞出。

\section{2 微滴喷射过程建模}

为了研究直接驱动型压电式喷头微滴喷射的成 形过程, 利用多物理场仿真平台, 根据包含表面张 力的不可压缩纳维-斯托克斯方程建立流体控制方 程, 在仿真环境中建立喷头的二维轴对称模型, 采 用具有重新初始化的水平集法追踪两相流界面, 对 微滴喷射成形过程进行仿真研究。

\section{1 流体控制方程组}

根据流体力学理论, 在不影响模拟准确性的前提 下，对模型进行如下定义: 喷射材料为不可压缩的牛 顿流体, 流体在圆直管中为层流状态, 喷射过程中无 热传递及能量耗散。采用不可压缩纳维-斯托克斯方程 (包括表面张力) 描述流体之间质量和动量的关系

$$
\frac{\partial \rho}{\partial t}+\nabla \cdot \rho \boldsymbol{v}=0
$$

$$
\rho\left(\frac{\partial \boldsymbol{v}}{\partial t}+\boldsymbol{v} \cdot \nabla \boldsymbol{v}\right)=-\nabla p+\nabla \cdot \mu\left(\nabla \boldsymbol{v}+(\nabla \boldsymbol{v})^{\mathrm{T}}\right)+\rho \boldsymbol{g}+\boldsymbol{F}_{\boldsymbol{S} \boldsymbol{T}}
$$


式中, $\rho$ 为流体密度; $p$ 为压力; $\nabla$ 为哈密顿算子; $v$ 为流体流动的速度矢量; $\mu$ 为流体黏度; $F_{S T}$ 为体 积力。

模型中存在气液两相, 因此仿真中需要对流体 界面进行跟踪。采用具有重新初始化的水平集法追 踪两相流界面, 此方法的对流运输方程如下

$$
\frac{\partial \phi}{\partial t}+\boldsymbol{v} \cdot \nabla \phi+\gamma\left[\left(\nabla \cdot\left(\phi(1-\phi) \frac{\nabla \phi}{|\nabla \phi|}\right)\right)-\varepsilon \nabla \cdot \nabla \phi\right]=0 \text { (3) }
$$

式中, $\phi$ 为水平集数, $\phi$ 在气相中为 0 , 在液相中为 1 , 靠近界面的过渡层中在 0 和 1 中平滑过渡; $\gamma$ 和 $\varepsilon$ 是数值计算稳定性参数, 迁移率 $\gamma$ 决定重新初始 化量, $\varepsilon$ 决定界面厚度。

为了抑制式(2)求解过程中的数值振荡, 采用水平 集函数来平滑界面两侧不连续变化的两相密度和黏度

$$
\begin{aligned}
& \rho=\rho_{\text {air }}+\left(\rho_{\text {ink }}-\rho_{\text {air }}\right) \phi \\
& \mu=\mu_{\text {air }}+\left(\mu_{\text {ink }}-\mu_{\text {air }}\right) \phi
\end{aligned}
$$

微滴喷射过程中产生微滴需要克服表面张力, 为了获得准确液滴的速度和体积, 必须考虑表面张 力对微滴喷射的影响, 本文采用连续表面张力模型

$$
\boldsymbol{F}_{S T}=\sigma \delta \kappa \boldsymbol{n}
$$

式中, $\sigma$ 为表面张力系数; $\delta$ 为界面 Dirac delta 函 数, 仅在流体界面为非零值; $\kappa$ 为界面曲率; $\boldsymbol{n}$ 为 界面单位法向矢量。

气液两相界面处的曲率和单位法向矢量可以根 据水平集函数分别表示为

$$
\begin{gathered}
\kappa=-\nabla \cdot \boldsymbol{n} \\
\boldsymbol{n}=\frac{\nabla \phi}{|\nabla \phi|}
\end{gathered}
$$

为了简化表面张力的计算, $\delta$ 函数近似为

$$
\delta=6|\phi(1-\phi)||\nabla \phi|
$$

\section{2 微滴喷射的有限元模型建立}

为了获得直接驱动型压电式喷头喷射过程微滴 自由表面的形状及位置, 跟踪微滴成形过程, 建立 喷头的多物理场二维轴对称模型。由于电直接驱动 微滴成形过程中涉及压电、固液、两相流多场耦合, 直接耦合难度大, 研究中多采用间接耦合法 ${ }^{[16-17]}$, 基 于此, 本节通过分别构建压电耦合模型、固液耦合 模型及微滴喷射的两相流模型, 实现喷头模型的建 立, 如图 3 所示, 采用 I、II、III 分别表示液相区、 压电陶瓷、气相区, 采用 $A 、 B 、 C 、 \cdots 、 N$ 对边界 进行标号。

压电耦合模型是为了获得环形压电陶瓷在驱动 电压作用下内壁产生的径向位移, 仿真模型中, 为

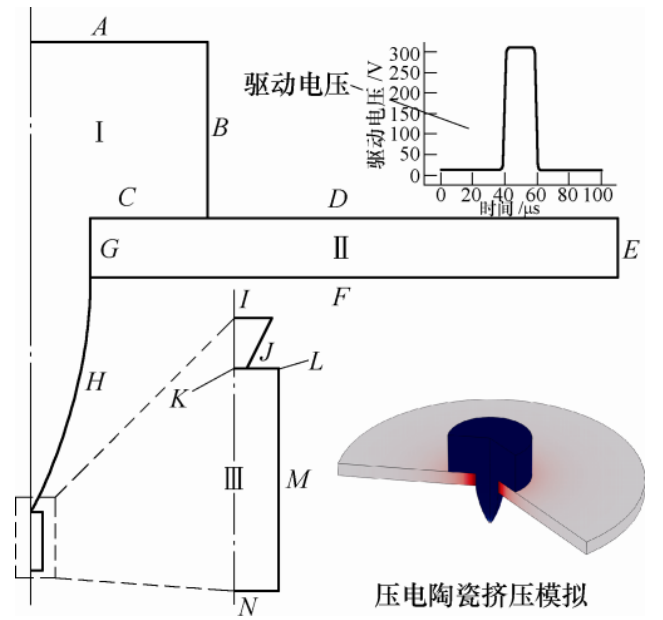

图 3 微滴喷射模型

与试验参数相对应, 环形压电陶瓷外径为 $20 \mathrm{~mm}$, 内径为 $2 \mathrm{~mm}$, 厚度为 $1 \mathrm{~mm}$, 陶瓷材料为 PZT-5 压电材料, 坐标系定义为 $Z X$ 平面系统, 辊约束 $D$ 、 $F$ 边界, $G 、 E$ 边界为自由边界, $F$ 边界接地, $D$ 边 界加载脉冲激励信号, 连续采集 $G$ 边界的位移量, 得到最大变形量和驱动电压的关系, 如图 4 所示。

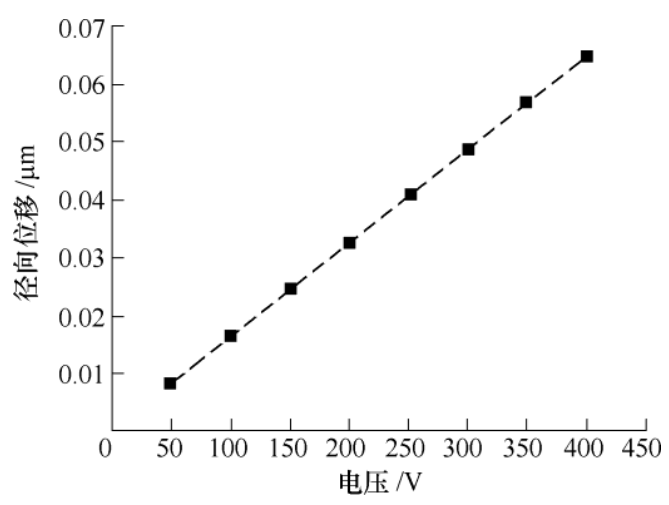

图 4 驱动电压与最大径向形变的关系

图 4 中, 陶瓷内壁的最大径向位移与驱动电压 的幅值基本成线性变化, 在忽略其他因素的前提下, 增大驱动电压幅值, 能得到较大的收缩位移。

固液耦合模型研究环形压电陶瓷与喷射材料之 间的相互作用, 将压电耦合模型中 $G$ 边界的位移作 为边界条件加载到固液耦合模型上, 建立与流固耦 合模块的全耦合仿真模型, 仿真模型中, 边界 $A$ 为液体入口、长度 $3 \mathrm{~mm}$, 边界 $B$ 长度 $3 \mathrm{~mm}$, 边界 $C$ 长度 $2 \mathrm{~mm}$, 喷嘴长度 $2.9 \mathrm{~mm}$, 边界 $I$ 为 液体出口、长度 $0.1 \mathrm{~mm}$, 边界 $B 、 C 、 H$ 为无滑 移壁面, 模型采用自由四边形网格划分, 求解器 采用全耦合求解器, 求解模型得到喷射材料在边 界 $I$ 的出口速度。

两相流模型研究微滴喷射中气相和液相分布的 情况, 即微滴喷射成形的过程, 通过将固液耦合模 型的边界 $I$ 的出口速度设置为两相流模型的入口速 
度, 设置区域 I 为喷射材料, 区域 III 为空气, 边界 $K$ 为喷射材料和空气的初始界面、长度 $50 \mu \mathrm{m}$, 边 界 $M$ 长度 $1 \mathrm{~mm}$, 边界 $N$ 为速度出口、长度 $0.2 \mathrm{~mm}$, 边界 $J 、 L 、 M$ 为无滑移壁面, 模型采用具有重新初 始化的水平集法对流体界面进行追踪, 采用自适应 网格细化, 求解器采用全耦合求解器。

\section{3 微滴成形过程}

为了验证建立的多物理场耦合模型可实现微滴 的按需喷射, 设置喷射材料黏度为 $20 \mathrm{mPa} \cdot \mathrm{s}$ 、表 面张力为 $0.07 \mathrm{~N} / \mathrm{m}$, 驱动电压幅值为 $250 \mathrm{~V}$ 、脉冲 宽度为 $20 \mu \mathrm{s}$, 得到微滴喷射成形过程液相图, 如图 5 所示。

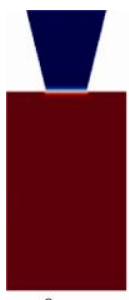

$0 \mu \mathrm{s}$

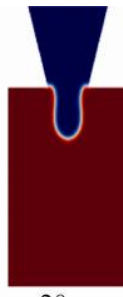

$20 \mu \mathrm{s}$

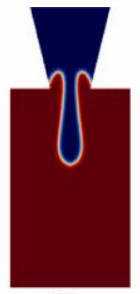

$40 \mu \mathrm{s}$

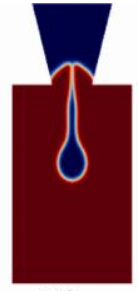

$110 \mu \mathrm{s}$

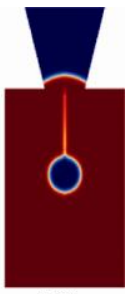

$160 \mu \mathrm{s}$
图 5 液滴喷射成形过程

由图 5 知, 微滴成形过程主要经历了微滴形成 液柱、颈缩、断裂、脱落四个阶段。 $0 \sim 20 \mu \mathrm{s}$ 为液 柱形成及生长阶段, 环形压电陶瓷在驱动电压的作 用下收缩变形直接挤压喷射材料, 喷射材料从喷嘴 口外凸形成液柱并开始伸长，20 110 $\mu \mathrm{s}$ 进入颈缩 阶段, 随着压电陶瓷的复位, 喷腔体积增大内部压 力减小, 喷嘴口处的液柱速度减小, 而液柱前端在 惯性的作用下继续向下运动, 在液柱尾部出现颈缩。 $110 \sim 160 \mu \mathrm{s}$ 为液柱断裂及微滴脱落阶段, 随着颈 缩的加剧, 液柱尾部发生断裂, 断裂的液体脱离喷 嘴形成液滴，在表面张力和惯性的共同作用下呈近 似球形飞出。可以看出, 所建模型可以实现微滴成 形过程的仿真模拟。

\section{3 模拟结果及分析}

均匀微滴稳定喷射主要由驱动电压大小及材料 的本身属性决定, 其中驱动电压的幅值大小决定压 电陶瓷的形变程度, 进而影响喷嘴容腔的体积变化, 是微滴喷射成形的关键。喷射材料的黏度及表面张 力对微滴喷射成形的稳定性有很大的影响 ${ }^{[18]}$ 。基于 此, 本节针对驱动电压、喷射材料黏度及表面张力 对微滴成形的影响进行研究。

\section{1 电压幅值对微滴成形的影响}

为了研究驱动电压幅值对微滴成形过程的影 响, 设定喷射材料的黏度 $20 \mathrm{mPa} \cdot \mathrm{s}$ 、表面张力为
$0.07 \mathrm{~N} / \mathrm{m}$, 依次在驱动电压幅值为 $150 \mathrm{~V} 、 200 \mathrm{~V}$ 、 $250 \mathrm{~V} 、 300 \mathrm{~V} 、 350 \mathrm{~V}$ 下对微滴成形过程进行模拟 仿真, 得到不同电压下微滴在 $110 \mu \mathrm{s}$ 时的液相分布 图, 如图 6 所示。

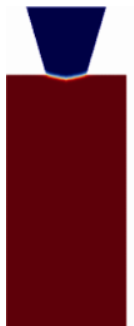

$150 \mathrm{~V}$

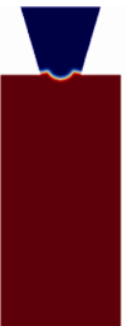

$200 \mathrm{~V}$

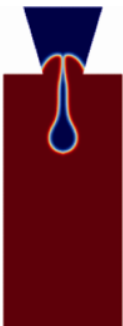

$250 \mathrm{~V}$

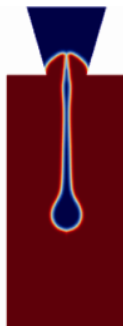

$300 \mathrm{~V}$

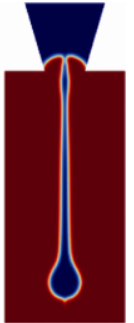

$350 \mathrm{~V}$
图 6 不同电压下微滴喷射成形过程

由图 6 可知, 驱动电压小于 $250 \mathrm{~V}$ 时, 喷出的 液柱未形成断裂, 液滴不能喷出; 当驱动电压在 $250 \mathrm{~V}$ 以上时, 可形成单独脱落的微滴, 电压过大 时会在断裂阶段液滴尾部会造成较长拖拽。为了明 确不同驱动电压对微滴喷出的速度及成形微滴体积 的影响规律，连续采集喷嘴中心点的喷射材料速度 及喷出液体的体积，如图 7、8 所示。

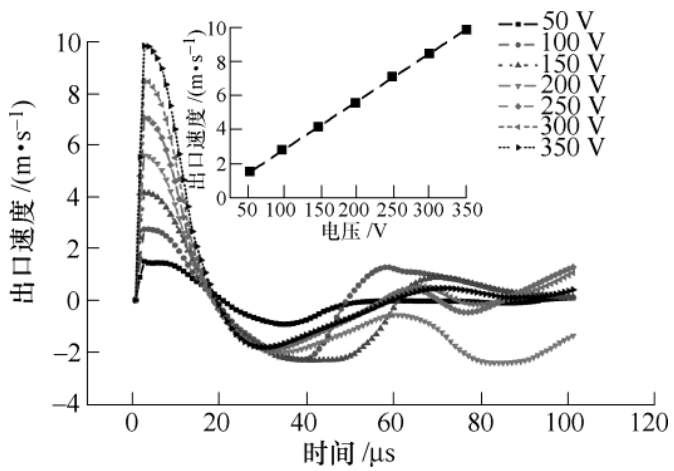

图 7 不同电压下的喷嘴口速度

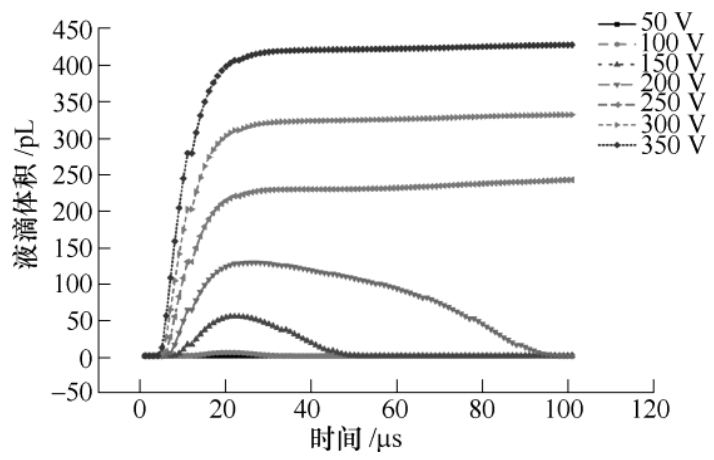

图 8 不同电压下喷射液滴的体积

从图 7 可以看出, 随着驱动电压幅值的增加, 喷嘴处喷出液柱的速度增大, 出口速度与驱动电压 的幅值成线性变化, 到达最大值后速度下降并出现 振荡, 最终在出口处速度趋于零。图 8 中, 当驱动 电压大于 $250 \mathrm{~V}$ 时, 喷出液体的体积达到最大值后 
保持不变, 这是由于此时的液滴已经脱离喷嘴, 形 成微滴飞出。而 $250 \mathrm{~V}$ 以下时, 喷出的液体体积到 达最大值后开始逐渐减小, 最终变为零, 这是由于 驱动力的不足, 喷出的液柱被重新吸回了喷嘴。

\section{2 液体黏度对微滴成形的影响}

设置驱动电压幅值为 $250 \mathrm{~V}$, 流体的表面张力为 $0.07 \mathrm{~N} / \mathrm{m}$, 流体黏度分别为 $1 \mathrm{mPa} \cdot \mathrm{s} 、 10 \mathrm{mPa} \cdot \mathrm{s}$ 、 $20 \mathrm{mPa} \cdot \mathrm{s} 、 30 \mathrm{mPa} \cdot \mathrm{s} 、 40 \mathrm{mPa} \cdot \mathrm{s}$ 进行喷射过程 仿真研究, 得到不同黏度下 $110 \mu \mathrm{s}$ 时微滴的液相分 布图, 如图 9 所示。

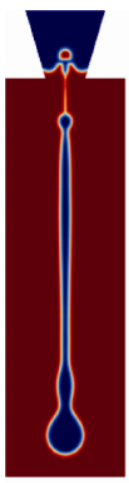

$0 \mu \mathrm{s}$

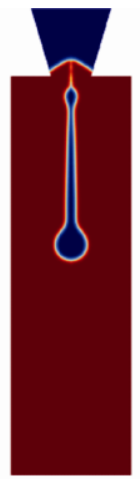

$20 \mu \mathrm{s}$

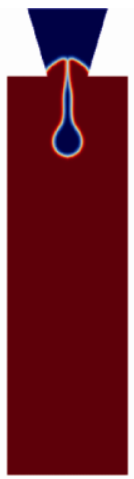

$40 \mu \mathrm{s}$

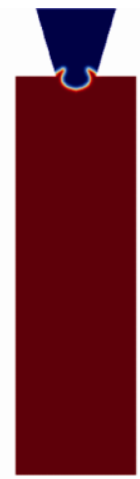

$110 \mu \mathrm{s}$

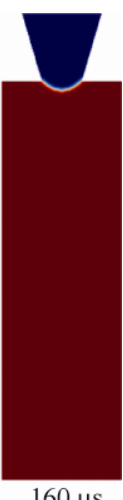

$160 \mu \mathrm{s}$
图 9 不同黏度下微滴喷射成形过程

由图 9 可知, 当喷射材料黏度小于 $20 \mathrm{mPa} \cdot \mathrm{s}$ 时, 可形成脱落的液滴, 但黏度较小时 $(1 \mathrm{mPa} \cdot \mathrm{s})$, 脱落的液滴将有较长拖拽, 同时低黏度的液体会在 喷嘴口处卷吸空气, 在喷头中形成气泡; 当黏度大 于 $30 \mathrm{mPa} \cdot \mathrm{s}$ 时, 液体获得的驱动力不足以克服黏 滞阻力及表面张力, 无法形成单独的微滴, 且随着 喷射材料黏度的增大, 微滴的断裂点离喷嘴的距离 就越近。为了进一步分析喷射材料黏度对微滴喷出 的速度及成形微滴体积的影响规律, 通过对喷嘴口 液柱最大速度及喷出喷嘴的液体体积进行采集, 得 到不同黏度下微滴成形过程中速度及微滴体积, 如 图 10、11 所示。

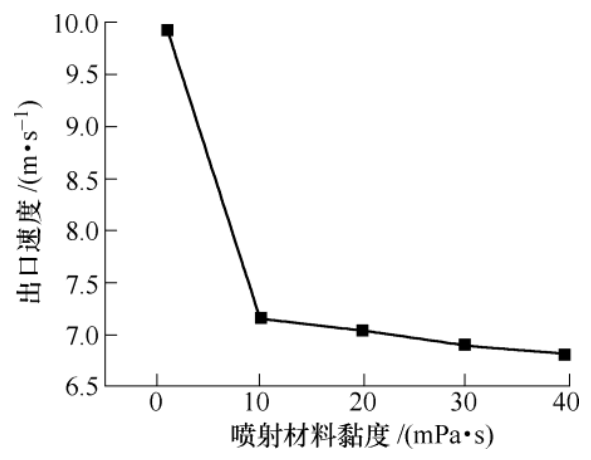

图 10 喷射材料黏度与出口速度的关系

从图 10 可以看出, 随着喷射材料黏度的增加, 微滴喷出的速度将减小, 黏度为 $1 \mathrm{mPa} \cdot \mathrm{s}$ 的喷射
材料在喷嘴处最大喷射速度可到 $10 \mathrm{~m} / \mathrm{s}$, 当黏度在 $10 \mathrm{mPa} \cdot \mathrm{s}$ 以上时速度变化趋势不大。图 11 中, 随 着喷射材料黏度的增加, 喷出微滴的体积将减小, 黏度为 $1 \mathrm{mPa} \cdot \mathrm{s}$ 的喷射材料可产生体积约 $800 \mathrm{pL}$ 的微滴。

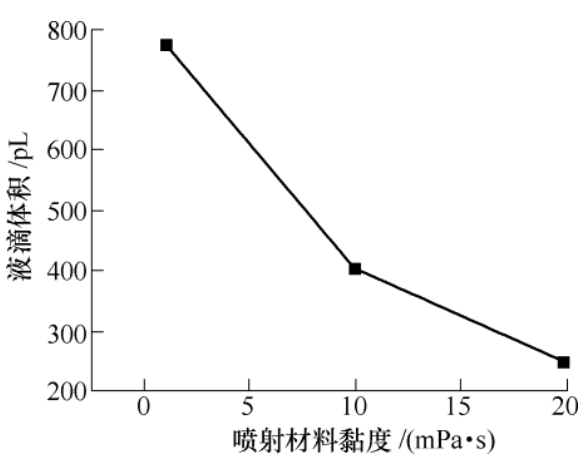

图 11 喷射材料黏度与微滴体积的关系

\section{3 液体表面张力对微滴成形的影响}

设置驱动电压幅值为 $100 \mathrm{~V}$, 黏度为 $1 \mathrm{mPa} \cdot \mathrm{s}$, 对喷射材料表面张力分别为 $0.02 \mathrm{~N} / \mathrm{m} 、 0.05 \mathrm{~N} / \mathrm{m}$ 、 $0.07 \mathrm{~N} / \mathrm{m} 、 0.09 \mathrm{~N} / \mathrm{m} 、 0.11 \mathrm{~N} / \mathrm{m}$ 进行喷射过程仿真 研究, 得到不同表面张力喷射材料在 $110 \mu \mathrm{s}$ 时微滴 的液相分布图，如图 12 所示。

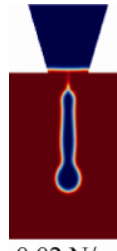

$0.02 \mathrm{~N} / \mathrm{m}$

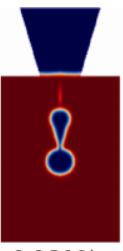

$0.05 \mathrm{~N} / \mathrm{m}$

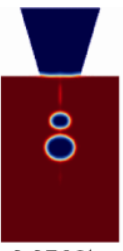

$0.07 \mathrm{~N} / \mathrm{m}$

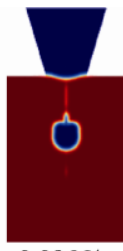

$0.09 \mathrm{~N} / \mathrm{m}$

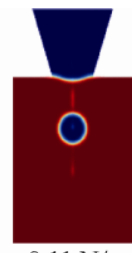

$0.11 \mathrm{~N} / \mathrm{m}$
图 12 不同表面张力下微滴喷射成形过程

由图 12 可知, 随着喷射材料表面张力的增加, 液滴拖拽长度减小，卫星滴消失，且液滴形态更易 成球形。为了明确喷射材料表面张力对微滴喷出的 速度及成形微滴体积的影响规律，对喷嘴液柱最大 速度及喷出喷嘴的液体体积进行采集，得到不同表 面张力下微滴成形过程中喷嘴处的速度及微滴的体 积，如图 13、14 所示。

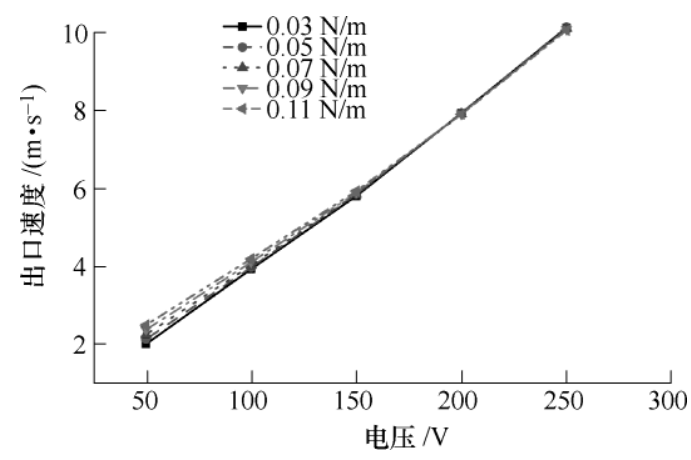

图 13 不同表面张力下微滴喷射速度 


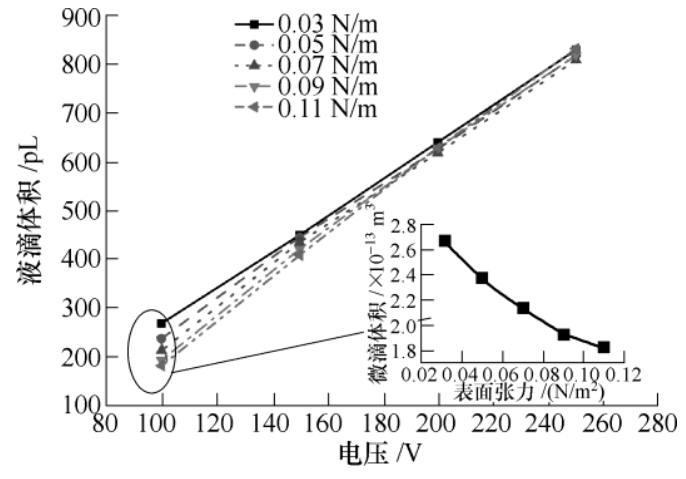

图 14 不同表面张力下微滴喷射体积

从图 13 可以看出, 随着驱动电压的增大, 液体 表面张力对喷头喷嘴处速度的影响减弱, 当驱动电 压在 $200 \mathrm{~V}$ 以上时, 材料的表面张力几乎不对喷嘴 口液柱速度造成影响。图 14 中, 在驱动电压 $100 \mathrm{~V}$ 时, 随着表面张力的增大, 喷射出的液体体积将减 小, 但当驱动电压增大时, 喷射出的液滴体积间的 差异将越来越小。

\section{4 微滴喷射试验}

为了对直接驱动型压电式喷头的仿真结果进行 验证, 利用搭建的微滴喷射实验系统进行喷射试验 研究, 构建的微滴喷射系统主要由驱动电源、压电 式喷头、示波器、图像采集系统等组成, 如图 15 所示。其中, 驱动电源可产生电压幅值 $0 \sim 400 \mathrm{~V}$ 、 频率 $0 \sim 30 \mathrm{~Hz}$ 、脉冲宽度 $10 \sim 50 \mu \mathrm{s}$ 的驱动电压; 压电式喷头由玻璃喷嘴、玻璃储液腔及环形压电陶 瓷驱动元件组成, 三者通过环氧树脂粘合, 喷嘴口 的直径为 $65 \mu \mathrm{m}$; 示波器用于观测产生的驱动电压; 图像采集系统为奥林巴斯 i-SPEED2 高速相机, 设 置帧率 100000 帧/s, PC 端可对微滴喷射图像进行 观测和储存。为了与试验过程进行对比, 将模型中 喷嘴直径设定为 $65 \mu \mathrm{m}$ 。

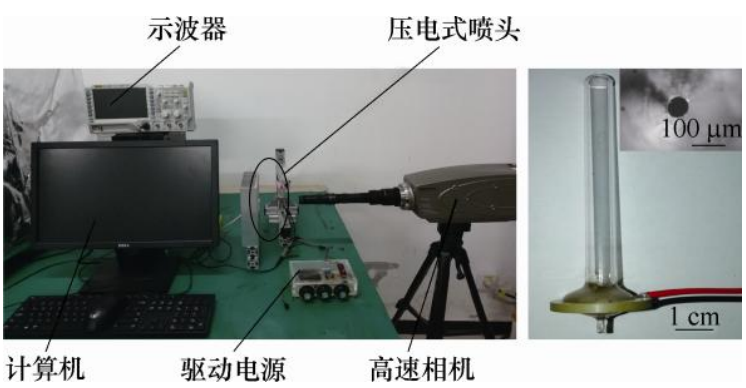

图 15 试验装置及压电式喷头

本次试验喷射材料为去离子水 (密度 $1.0 \mathrm{~g} / \mathrm{cm}^{3}$ 、 黏度 $1 \mathrm{mPa} \cdot \mathrm{s}$ 、表面张力 $0.073 \mathrm{~N} / \mathrm{m}$ ), 在驱动电压 幅值 $200 \mathrm{~V}$ 、频率 $1 \mathrm{~Hz}$ 、脉冲宽度 $10 \mu \mathrm{s}$ 的驱动信
号作用下, 得到微滴成形过程如图 16a 所示, 仿真 结果如图 16b 所示。
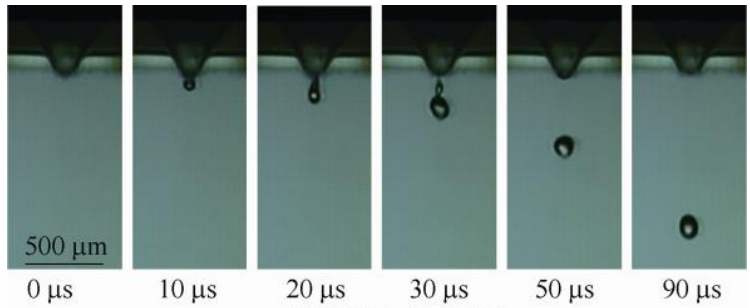

(a) 微滴实际成形过程

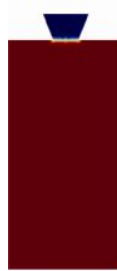

$0 \mu \mathrm{s}$

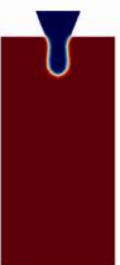

$10 \mu \mathrm{s}$

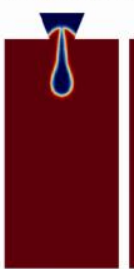

$20 \mu \mathrm{s}$

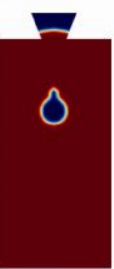

$30 \mu \mathrm{s}$

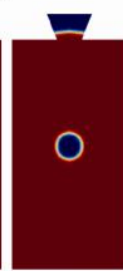

$50 \mu \mathrm{s}$

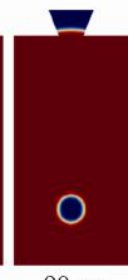

$90 \mu \mathrm{s}$

(b) 微滴模拟成形过程

图 16 液滴实际成形过程及模拟过程对比

从图 16a 可以看出微滴喷射稳定, 无卫星滴, 成形液滴直径在 $80 \mu \mathrm{m}$ 左右。与图 $16 \mathrm{~b}$ 的模拟结果 对比可以看出, 模拟结果与实际微滴成形过程吻合 较好, 表明设计的直接驱动型压电式喷头在控制信 号作用下，可按需产生均匀微滴。

\section{5 结论}

（1）设计了直接驱动型压电式喷头，建立了基 于多物理场耦合的压电式喷头微滴喷射模型(包括 压电耦合模块、固液耦合模块、两相流模块)，模拟 了微滴成形过程。

(2) 研究了驱动电压、液体黏度及表面张力等 因素对微滴成形过程的影响, 驱动电压的幅值是决 定微滴喷射的关键, 驱动电压幅值与喷射微滴的速 度及体积呈近线性关系，黏度增大会降低微滴的喷 射速度及成形微滴的体积，表面张力会影响液滴的 拖拽长度及喷出微滴的形状。

（3）搭建了微滴喷射实验平台, 对直接驱动型 压电式喷头喷射微滴进行了初步试验，验证了直接 驱动型压电式喷头能够实现微滴的有效喷射，喷射 试验结果与仿真较好吻合。

\section{参 考 文 献}

[1] NGO T D, KASHANI A, IMBALZANO G, et al. Additive manufacturing (3D printing): A review of materials, methods, applications and challenges[J]. Composites Part B: Engineering, 2018, 143: 172-196. 
[2] VLĂSCEANU G M, IOVU H, IONIȚĂ M. Graphene inks for the $3 \mathrm{D}$ printing of cell culture scaffolds and related molecular arrays[J]. Composites Part B : Engineering, 2019, 162: 712-723.

[3] SOCHOL R D, SWEET E, GLICK C C, et al. 3D printed microfluidics and microelectronics[J]. Microelectronic Engineering, 2018, 189: 52-68.

[4] 齐乐华, 钟宋义, 罗俊. 基于均匀金属微滴喷射的 3D 打印技术[J]. 中国科学: 信息科学, 2015,45(2): 212-223. QI Lehua, ZHONG Songyi, LUO Jun. Three- dimensional printing technology based on uniform metal droplet ejecting[J]. Scientia Sinica Informationis，2015，45(2): 212-223.

[5] 肖波, 吴姗, 刘金玲, 等. 织物表面微滴喷射反应成形 导电线路基础研究 [J]. 机械工程学报, 2018, 54(7): 216-222.

XIAO Yuan, WU Shan, LIU Jinling, et al. Basic research on conductive lines by droplets jet and reaction forming on fabric surface[J]. Journal of Mechanical Engineering, 2018, 54(7): 216-222.

[6] LU X, ZHAO T, JI X, et al. 3D printing well organized porous iron-nickel/polyaniline nanocages multiscale supercapacitor[J]. Journal of Alloys and Compounds, 2018, 760: 78-83.

[7] THABET Y, BREITKREUTZ J. Printing pharmaceuticals by inkjet technology: Proof of concept for stand-alone and continuous in-line printing on orodispersible films[J]. Journal of Manufacturing Processes, 2018, 35: 205-215.

[8] GOGHARI A A, CHANDRA S. Producing droplets smaller than the nozzle diameter by using a pneumatic drop-on-demand droplet generator[J]. Experiments in Fluids, 2008, 44(1): $105-114$.

[9] 舒霞云, 张鸿海. 多材料按需微滴喷射系统设计与实验 研究[J]. 机械科学与技术, 2015，34(2)：257-262.

SHU Xiayun, ZHANG Honghai. Design and experimental study on droplet-on-demand jetting system for multi-materials[J]. Mechanical Science and Technology for Aerospace Engineering， 2015，34(2): 257-262.

[10] 仲作金, 褚祥诚, 陈海超, 等. 瓷砖装饰用压电喷墨打 印头关键技术研究 [J]. 压电与声光, 2016, 38(2): 221-226.
ZHONG Zuojin, CHU Xiangheng, CHEN Haichao, et al. A research review on the key technologies of piezoelectric inkjet printhead for tile decoration[J]. Piezoelectric \& Acoustooptics, 2016, 38(2): 221-226.

[11] OBROVAC M N, CHRISTENSEN L, LE D B, et al. Alloy design for lithium-ion battery anodes[J]. Journal of The Electrochemical Society, 2007, 154(9): A849-A855.

[12] 杨明明, 汪焰恩, 魏生民, 等. 径向极化压电陶瓷管建模 与仿真 $[J]$. 电子元件与材料, 2018，37(1): 23-27, 44. YANG Mingming, WANG Yanen, WEI Shengmin, et al. Modeling and simulation of radial polarized piezo tube [J]. Electronic Components and Materials, 2018, 37(1): $23-27,44$

[13] WU H C, LIN H J, HWANG W S. A numerical study of the effect of operating parameters on drop formation in a squeeze mode inkjet device[J]. Modelling and Simulation in Materials Science and Engineering, 2004, 13(1): 17.

[14] RONE W S, BEN-TZVI P. MEMS-based microdroplet generation with integrated sensing $[\mathrm{C}] / /$ Proc. COMSOL Conf. 2011.

[15] RONE W, BEN-TZVI P. Design and FE analysis of integrated sensing using gas compressibility for microdroplet generation[J]. Mechatronics, 2013, 23(4) : 397-408.

[16] WIJSHOFF $\mathrm{H}$. The dynamics of the piezo inkjet printhead operation[J]. Physics Reports, 2010， 491(4-5): 77-177.

[17] 范增华, 荣伟涁, 王乐锋, 等. 压电驱动微点胶器的控 制与实验 $[J]$. 光学精密工程, 2016，24(5): 1042-1049. FAN Zenghua, RONG Weibin, WANG Lefeng, et al. Control and experiment of micro-dispenser by piezoelectric drive[J]. Optics and Precision Engineering, 2016, 24(5): 1042-1049.

[18] 占红武, 胥芳, 郭维锋, 等. 压电喷墨过程动力学建模 与供墨方法[J]. 机械工程学报, 2017，53(1): 140-149. ZHAN Hongwu, XU Fang, GUO Weifeng, et al. Dynamics modeling and ink supply methods of piezoelectric ink-jetting process[J]. Journal of Mechanical Engineering, 2017, 53(1): 140-149.

作者简介: 肖洲, 男, 1975 年出生, 博士, 教授, 硕士研究生导师。主 要研究方向微滴喷射打印、智能纺织品。

E-mail: xiaoyuanjidian@xpu.edu.cn 